W. Randerath

C. Rocholl

F. Feldmeyer

W. Galetke

\section{Quantifizierung der Druckvariabilität unter kontinuierlicher Positivdruckatmung bei obstruktivem Schlafapnoesyndrom}

Variability of the Treatment Pressure under Continuous Positive Airway Pressure Treatment in Obstructive Sleep Apnea Syndrome

\section{Zusammenfassung}

Hintergrund: Automatische CPAP-Verfahren werden derzeit neben der Bilevel-Therapie oder der nichtinvasiven Beatmung eingesetzt, wenn die konstante CPAP-Therapie insuffizient ist. Je nach Schlafstadium und Körperlage ist eine Variabilität des Druckbedarfes bekannt, die jedoch auch davon unabhängig bei der Analyse der nächtlichen Druckprofile beobachtet wurde. Mit dieser Studie sollte die Druckvariabilität quantifiziert und ihre Bedeutung beim obstruktiven Schlafapnoe-Syndrom (OSAS) ermittelt werden. Methode: Wir analysierten das Druckprofil einer 6-wöchigen Therapie mit impedanzgesteuertem automatischem CPAP $\left(\mathrm{APAP}_{\mathrm{FOT}}\right)$. Der Variabilitätsindex (VI) wurde aus den Abweichungen des Druckes vom Mittelwert ermittelt. Eine geringe Variabilität lag vor, wenn der VI im Mittel und in $\leq 10 \%$ der Einzelnächte $\leq 0,75$ lag. Patienten: 20 Patienten (Alter $55,5 \pm 10,9$ Jahre, BMI 36,6 $\pm 26,5 \mathrm{~kg} / \mathrm{m}^{2}$, AHI 36,9 $\pm 26,3 / \mathrm{h}$ ) wurden wegen CPAP-Intoleranz/-Ineffektivität mit $\mathrm{APAP}_{\mathrm{FOT}}$ behandelt. Ergebnisse: Bei suffizienter Therapie lag der VI lag im Mittel ( 6 Wochen) bei 0,9 $\pm 0,7$. Bei $50 \%$ der Patienten blieb der VI unter 0,75. Bei 7 dieser Patienten überschritt er den Grenzwert jedoch in mehr als 10\% der Nächte. Nur 3/20 Patienten erfüllten beide Kriterien der Druckstabilität. Der VI korrelierte signifikant mit dem mittleren Therapiedruck (r: 0,66, p < 0,001). Schlussfolgerungen: Eine ausgeprägte Variabilität des Druckbedarf findet sich regelhaft bei Patienten mit obstruktivem Schlafapnoe-Syndrom.

\section{Abstract}

Background: Selfadjusting CPAP therapy is mostly employed if constant CPAP does not sufficiently suppress respiratory disturbances or is not accepted by the patient. The number of respiratory disturbances and thus the pressure need varies with sleep stages and body position during sleep. However, the analysis of the pressure profile during automatic CPAP therapy indicates a relevant variability of treatment pressure which is independent of the above mentioned criteria. Therefore, we aimed to quantify the pressure variability and its significance in patients with obstructive sleep apnea syndrome (OSAS). Methods: We analysed the pressure profile during a six-week treatment period with selfadjusting CPAP therapy based on the measurement of the impedance $\left(\mathrm{APAP}_{\mathrm{FOT}}\right)$. The variability index $(\mathrm{VI})$ was calculated from the deviations of the treatment pressure from the mean pressure. The variability was considered to be low and clinically irrelevant if the VI did not exceed 0.75 as a mean value and in more than $10 \%$ of the nights. Patients: 20 patients (male 19, age $55.5 \pm 10.9$ years, BMI $36.6 \pm 26.5 \mathrm{~kg} / \mathrm{m}^{2}$, AHI $36.9 \pm 21.3 / \mathrm{h}$ ) who were treated with $\mathrm{APAP}_{\mathrm{FOT}}$ because of intolerance or inefficiency of constant CPAP. Results: The VI was $0.9 \pm 0.7$ (range $0.27 \pm 0.05$ to $1.95 \pm$ 0.83 ). The number of nights with a figure $\geq 0.75$ reached $17.6 \pm$ 13.8 (range $0-40$ ). In $50 \%$ of the patients the mean VI was lower than 0.75 . However, in 7 of these 10 patients the VI exceeded 0.75 in more than $10 \%$ of the nights $(4-15)$. Only 3 of 20 patients fulfilled both criteria of pressure stability. The VI showed a significant correlation with $\mathrm{P}_{\text {mean }}(\mathrm{r}: 0.66, \mathrm{p}<0.001)$. Conclusions: In most patients with difficult-to-treat OSAS a variability of the treatment pressure can be found. 
In der Therapie des obstruktiven Schlafapnoesyndroms (OSAS) hat sich die kontinuierliche Positivdruckatmung (CPAP) als effizientes Verfahren bewährt und stellt die Therapie der Wahl dar [1]. Auch wenn die CPAP-Behandlung von den meisten Patienten langfristig genutzt wird, zeigen doch $20-30 \%$ der Patienten eine unzureichende Akzeptanz und Compliance [2,3]. Um eine bessere Adaptation der Therapie an den Bedarf des Patienten zu ermöglichen, wurden automatische CPAP-Verfahren entwickelt, die den Druck während der gesamten Nacht, aber auch im Wechsel von Nacht zu Nacht und im Langzeitverlauf dem jeweiligen Obstruktionsgrad der oberen Atemwege anpassen [4-12]. Der mittlere Therapiedruck kann so signifikant gesenkt werden $[4,6,9-12]$. In weiten Teilen der Nacht werden nur die niedrigst möglichen Drücke appliziert, andererseits kann eine Reaktion auf Phasen sehr hohen Druckbedarfes erreicht werden [7]. Zur Zeit stehen zwei Verfahren zur Verfügung, die sensitiv zu messen und zu reagieren in der Lage sind. Sie basieren auf der Analyse der inspiratorischen Flusskontur sowie der Impedanz der oberen Atemwege mittels der forcierten Oszillationstechnik [4-11].

Das automatische CPAP-System auf dem Boden der forcierten Oszillationstechnik $\left(\mathrm{APAP}_{\mathrm{FOT}}\right)$ hat sich der konstanten CPAP-Therapie in der Suppression respiratorischer Störungen und der Verbesserung der Schlafqualität, einschließlich der Arousalaktivität, als gleichwertig erwiesen [9-11]. Es konnte auch gezeigt werden, dass bei schwierig zu behandelndem OSAS APAP ${ }_{\mathrm{FOT}}$ der in diesen Situationen häufig eingesetzten Bilevel-Therapie gleichwertig ist [12]. Sowohl im Vergleich zur konstanten CPAP-Therapie als auch im Vergleich zur Bilevel-Therapie zeigte sich eine deutliche Präferenz der Patienten zu Gunsten von APAP [10-12]. Automatische CPAP-Verfahren können somit durchaus als Standardtherapie neben konstanten CPAP eingesetzt werden [13], was jedoch vor allem aus ökonomischen Überlegungen noch nicht generell Eingang in die klinische Praxis gefunden hat. Zur Zeit gibt es noch keine allgemein akzeptierten Kriterien für die Anwendung automatischer CPAP-Verfahren. Die derzeitige Praxis verschiedener Arbeitsgruppen sieht vor, die automatische Behandlung bei den Patienten einzusetzen, bei denen eine wesentliche Abhängigkeit der respiratorischen Störungen von Körperlage und Schlafstadien besteht [11] oder bei denen eine Einstellung auf konstantes CPAP wegen einer unzureichenden Suppression der respiratorischen Störungen oder Non-Compliance nicht gelingt [12]. Eine weitere Indikation kann eine erhebliche Variabilität des Therapiedruckes innerhalb einer Nacht oder von Nacht zu Nacht sein. Es stellte sich daher die Frage, wie eine Quantifizierung dieser Variabilität möglich ist und bei welchem Anteil der Patienten eine Variabilität des Druckbedarfes nachzuweisen ist.

\section{Methodik}

Patienten: Wir untersuchten 20 Patienten (19 Männer, Alter $55,5 \pm 10,9$ Jahre, BMI $36,6 \pm 26,5 \mathrm{~kg} / \mathrm{m}^{2}$, AHI $\left.36,9 \pm 26,3 / \mathrm{h}\right)$, die zur Diagnostik und Therapie schlafbezogener Atemstörungen stationär aufgenommen wurden. Die Indikation zur automatischen CPAP-Therapie wurde gestellt, wenn mit konstantem CPAP eine suffiziente Beseitigung der respiratorischen Störungen nicht gelang oder diese nicht toleriert wurde.
Studiendesign: Nach polysomnographischem (PSG) Nachweis eines OSAS, manueller Titration eines konstanten CPAP und Einleitung einer Behandlung mit APAP $_{\mathrm{FOT}}$ (SomnoSmart ${ }^{\circledR}$, Weinmann, Hamburg) wurden die Patienten über sechs Wochen mit APAP $_{\mathrm{FOT}}$ (Druckspanne 4-18 mbar) behandelt. Die Patienten atmeten mit Standardmasken (Bubblemask ${ }^{\circledR}$, ResMed, Northright, Australien, SomnoMask $^{\circledR}$, Weinmann, Hamburg). Am Ende der Therapiephase erfolgte eine Kontrolle der PSG im Schlaflabor. Die Daten zur Compliance und zum Druckprofil wurden aus der Anwendungssoftware SomnoSupport ${ }^{\circledR}$ (Weinmann, Hamburg) ausgelesen.

Polysomnographie: Die PSG wurden nach den Richtlinien von Rechtschaffen und Kales und den ASDA-Kriterien [14,15] ausgewertet. Eine Apnoe wurde als Unterbrechung des Atemflusses für mehr als 10 Sekunden, eine Hypopnoe als Verminderung der Atemanstrengung um $\geq 50 \%$ über 10 Sekunden oder eine Verminderung der Atemanstrengung in Verbindung mit einer Sauerstoffdesaturation $u m \geq 4 \%$ angesehen.

Variabilitätsindex: Zur Quantifizierung der Variabilität des Druckbedarfes wurde in Anlehnung an frühere Untersuchungen ein Variabilitätsindex (VI) errechnet [16]. Dabei wurde berücksichtigt, in welchen relativen Zeitanteilen Abweichungen vom mittleren Behandlungsdruck vorliegen:

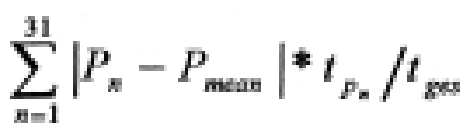

mit n $=1-31$, wobei $1 \cong$ Druckstufe $3,0-3,4$ mbar, $30 \cong 17,5-17,9$, $31 \cong \geq 18$ mbar. $P_{\text {mean }}$ : mittlerer Therapiedruck, $t_{P_{n}}$ : Zeitanteil $P_{n}$ $\mathrm{t}_{\mathrm{ges}}$ : Gesamtzeit.

Der VI kann Werte von 0 bis 7,5 annehmen. Ein VI von 0 liegt dann vor, wenn ein Patient während der gesamten Nacht auf einer Druckstufe atmet. Der VI erreicht den höchsten Wert, wenn in jeweils der Hälfte der Zeit die beiden Extreme der Druckstufen notwendig sind (50\% 3,0 mbar und 50\% 18 mbar). Werden alle Druckstufen in gleichem Maße durchlaufen, liegt der VI bei 3,9 (Abb.1a-d). Als Grenzwert für eine bedeutsame Variabilität wurde ein VI von 0,75 angesehen. Dieser Wert wird dann nicht überschritten, wenn der Druck um maximal drei mbar schwankt. Weiterhin ist nach unserer Definition die Variabilität dann als unbedeutend anzusehen, wenn der VI von 0,75 an nicht mehr als $10 \%$ der erfassten Nächte überschritten wird.

Statistik: Die Untersuchung auf signifikante Unterschiede mit $\mathrm{p}<0,05$ erfolgte mit dem Wilcoxon-Test. Beim Vergleich der nach dem $P_{\text {mean }}$ aufgeteilten Subgruppen wurde der Mann-Whitney-Test benutzt. Die Korrelation zwischen $P_{\text {mean }}$ und VI wurde mit der Spearman Rangkorrelation berechnet.

\section{Ergebnisse}

Die Patienten wurden über 40,5 $\pm 2,2$ Tage (Range 37-42) behandelt. Insgesamt wurden 809 Tage ausgewertet. Der AHI konnte unter $\mathrm{APAP}_{\mathrm{FOT}}$ von $36,9 \pm 21,3 / \mathrm{h}$ auf $8,7 \pm 4,1 / \mathrm{h}(\mathrm{p}<0,001)$ und die tiefste $\mathrm{O}_{2}$-Sättigung von $79,7 \pm 9,8 \%$ auf $86,9 \pm 4,2 \%$ $(p<0,001)$ verbessert werden. Dies ging mit einer Zunahme von 

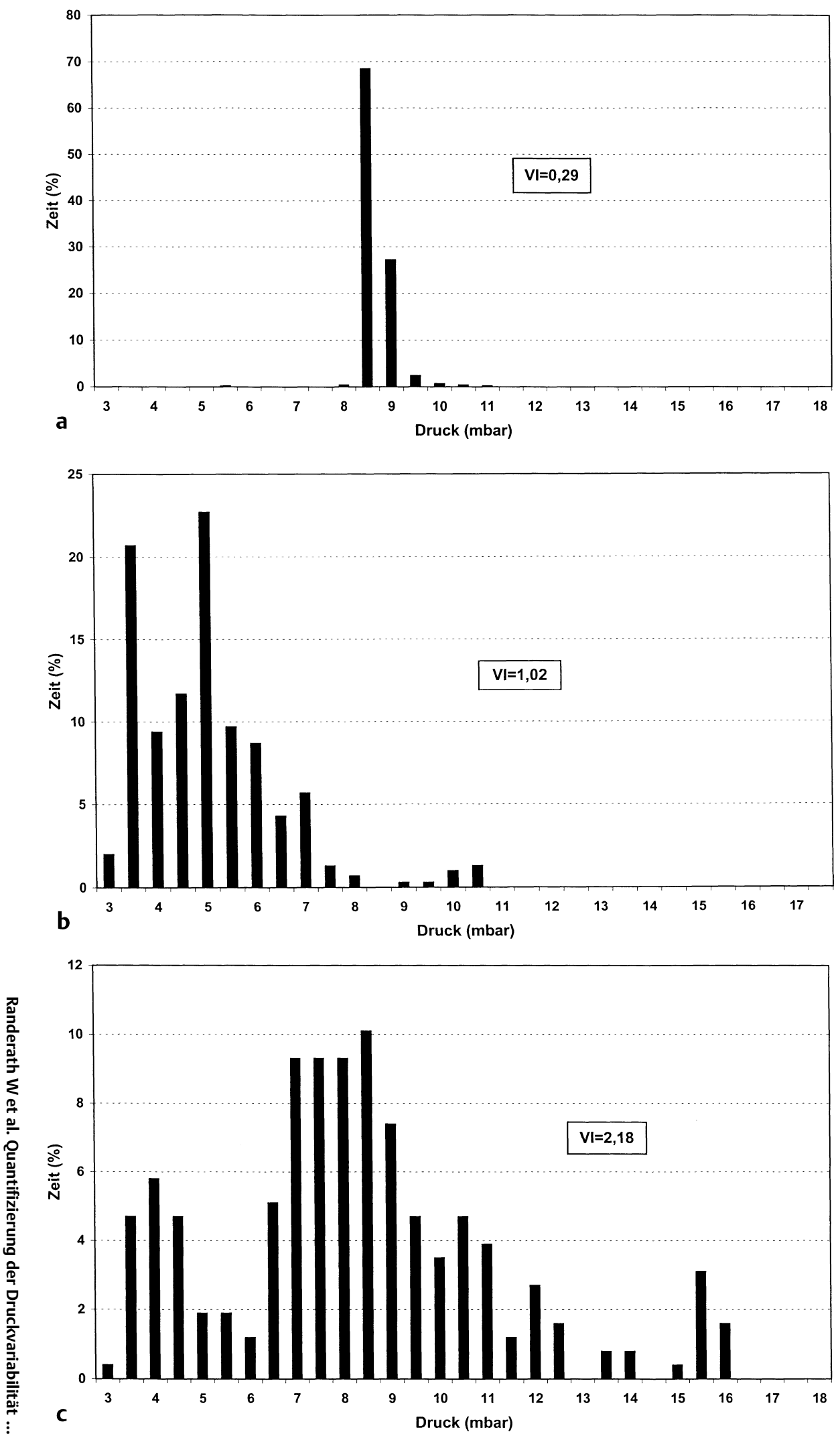

Abb. 1 a-d Beispiele des Variabilitätsindex (Einzelnächte)

a Hohe Druckstabilität: Die Grafik gibt die Zeitanteile (Ordinate) an, in denen die einzelnen Druckstufen appliziert wurden. In mehr als 95\% der Zeit lagen die Drücke zwischen 8,5 bis 9,9 mbar, der VI lag bei 0,29 und zeigte damit eine geringe Instabilität an. b Hohe Druckvariabilität: VI 1,02. 85\% der in dieser Nacht gemessenen Drücke streuen über vier mbar. Diese Streuung wird auch unabhängig von der übrigen Zeit, in denen die Drücke bereits außerhalb dieses Streubereiches liegen, als unzureichende Stabilität angesehen. c Hohe Streuung der Druckwerte: VI 2,18. Der Behandlungsdruck zeigt eine breite Streuung über ein Spektrum von neun mbar. Dabei sind die Zeitanteile annähernd gleich verteilt.
REM- und Tiefschlaf einher (REM vor Therapie: $12,1 \pm 5,1 \%$, unter Therapie: $15,8 \pm 7,4 \%$, n.s., S3/4 vor: $9,3 \pm 8,2 \%$, nach: $18,5 \pm$ $11,5 \%, \mathrm{p}<0,05)$. Die Nutzung der Therapie lag bei $5,7 \pm 2,3 \mathrm{~h} / \mathrm{d}$. Der über die gesamte Studiendauer gemittelte Behandlungsdruck $P_{\text {mean }}$ betrug 5,1 $\pm 1,3$ mbar, der maximale Druck 9,7 \pm 3,1 mbar, der minimale Druck 3,6 $\pm 1,4$ mbar. In 95\% der Zeit wurden Drücke $\leq 7,5 \pm 4,0$ mbar appliziert.
Der VI betrug über die gesamte Untersuchungszeit 0,9 $\pm 0,7$ (Abb. 2). Die Indices lagen bei den einzelnen Patienten zwischen $0,27 \pm 0,05$ und $1,95 \pm 0,83$. Die Anzahl der Nächte, in denen der VI von 0,75 überschritten wurde, lag bei 17,6 $\pm 13,8$ (Range 0 - 40) (Abb.3a-d). Bei 50\% der untersuchten Patienten lag der VI im Mittel der 6 Wochen unter 0,75. Allerdings fanden sich bei 7 dieser 10 Patienten mehr als 10\% [4 - 15] der Nächte, in denen der VI 


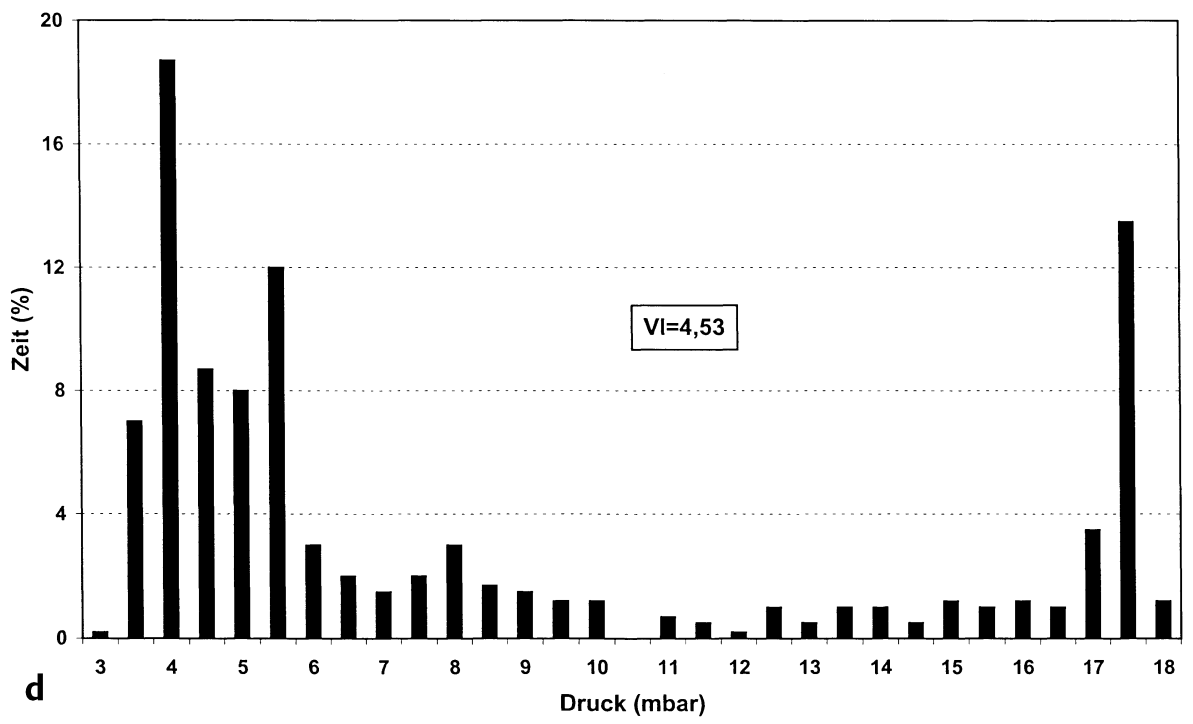

428
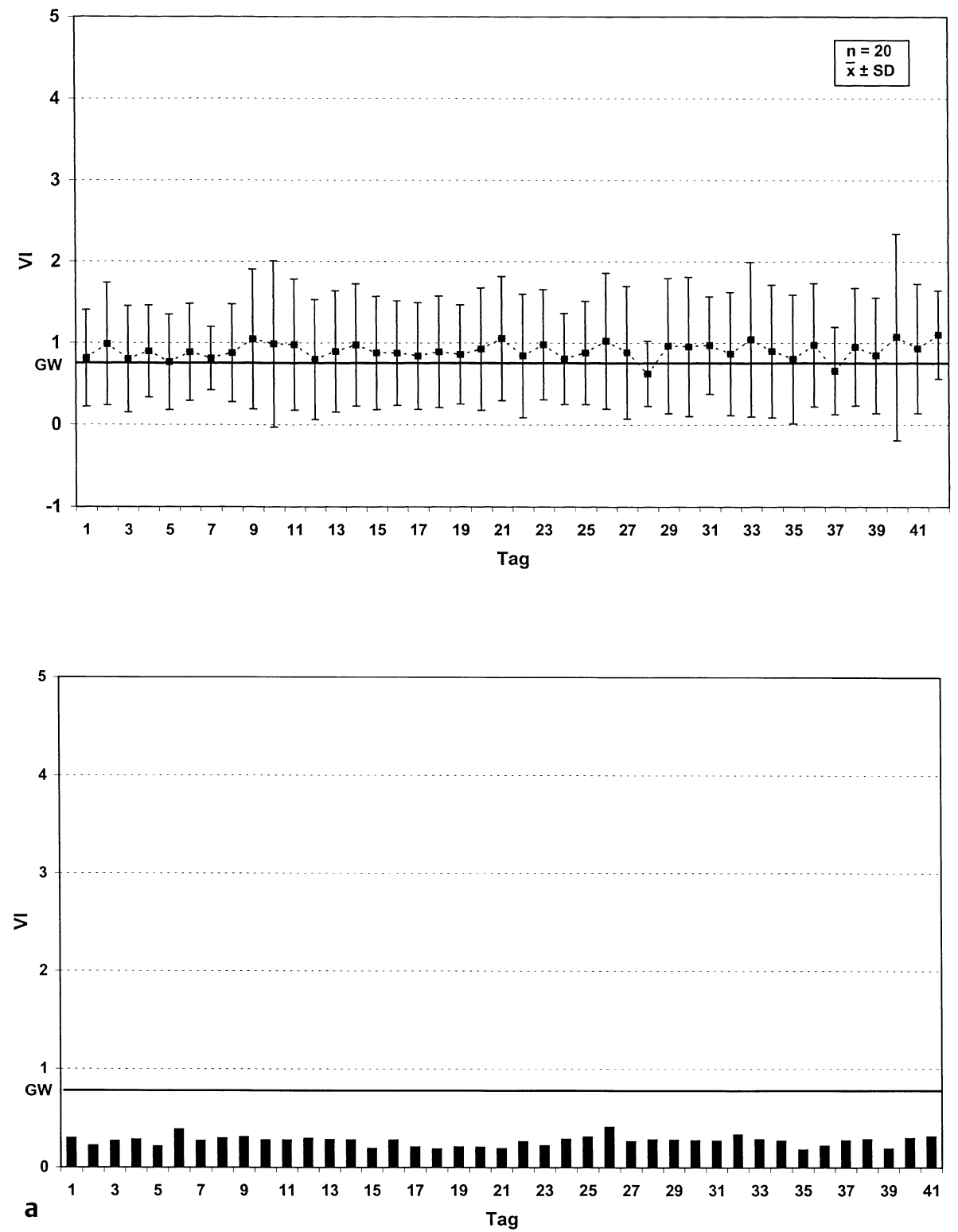

Abb. 1 d Extremwerte: VI 4,53. Die Verteilung der Drücke zeigt je einen Gipfel bei niedrigen und hohen Werten. Dies verdeutlicht die höchste Instabilität.

Abb. 2 Variabilitätsindex der Gesamtgruppe (6 Wochen) GW zeigt den Grenzwert von 0,75 an (durchgezogene Linie). Der VI liegt im Gesamtkollektiv regelhaft über dem GW, der eine Schwankung der Behandlungsdrücke von mehr als drei mbar anzeigt.

Abb. $\mathbf{3}$ a-d Verlauf des Variabilitätsindex im individuellen Verlauf

a Langfristig hohe Druckstabilität, VI regelhaft unter 0,75 . Indikation zur Therapie mit konstantem CPAP. 


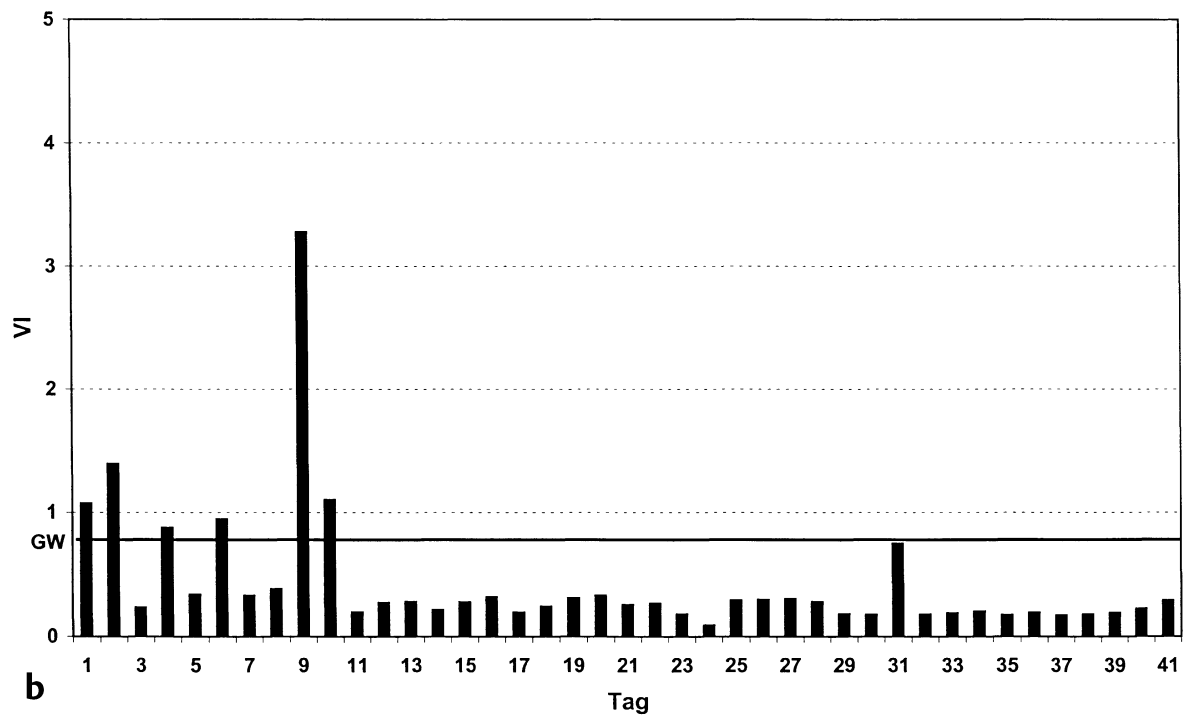

Abb. $\mathbf{3} \mathbf{b}-\mathbf{d}$

b In der Initialphase deutliche Instabilität des Druckbedarfes, die im Verlauf nachlässt (siehe dazu auch [15]).

c VI im 6-Wochen-Verlauf mit $0,83 \pm 0,66$ nur wenig über dem Grenzwert. Allerdings findet sich in 18 von 42 Nächten eine ausgeprägte Variabilität.

d Wechsel von stabilen und sehr instabilen Nächten. Die initiale Einstellung eines konstanten CPAP lässt eine unbefriedigende Behandlung in den meisten, sehr instabilen Folgenächten erwarten.
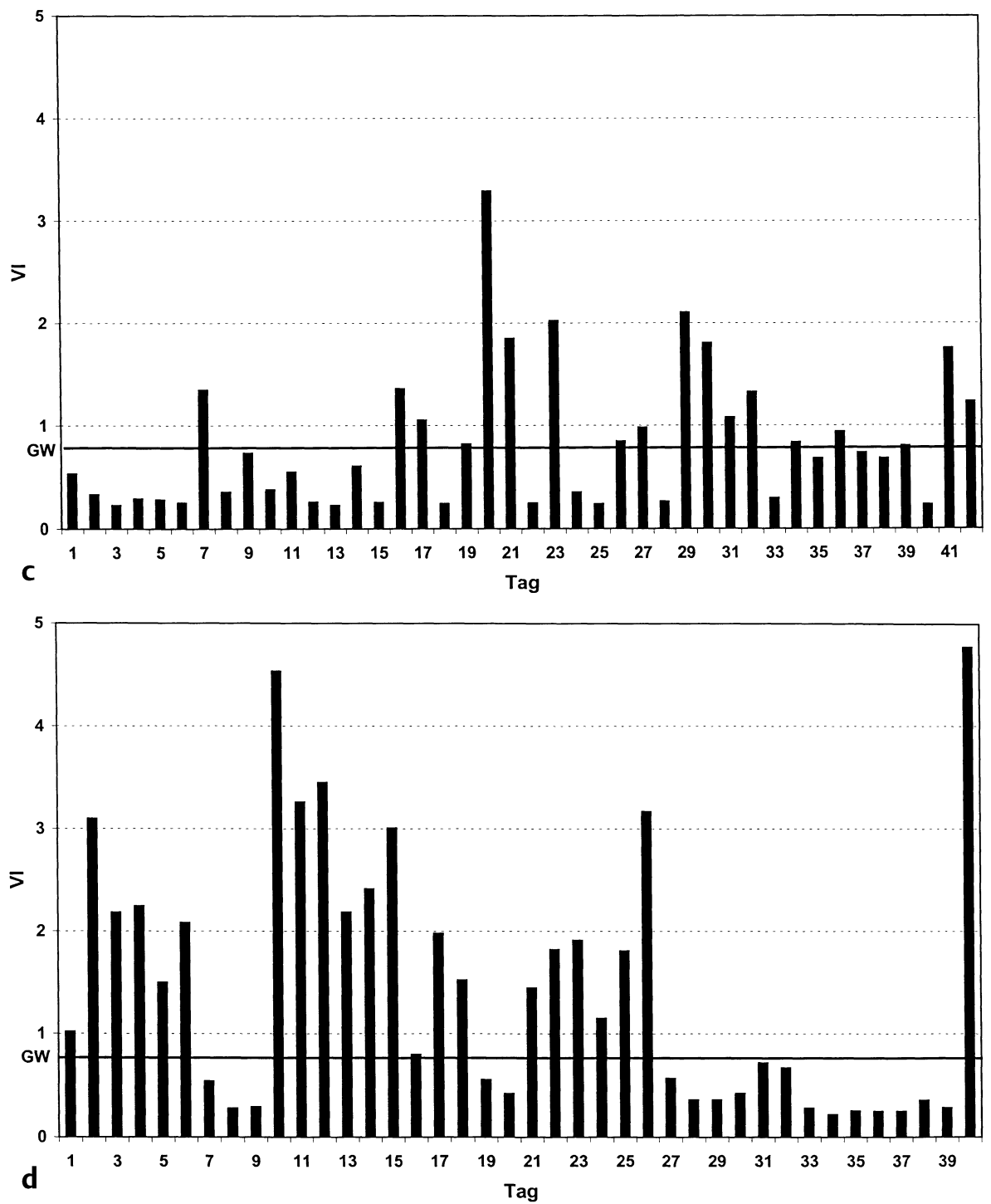

den Grenzwert von 0,75 überschritt. Somit erfüllten nur 3 der 20 Patienten beide Kriterien der Druckstabilität.

Um einen Einfluss des Therapiedruckes auf den VI zu untersuchen wurde das Kollektiv in zwei Subgruppen mit $\mathrm{P}_{\text {mean }}<5,1$
(Gruppe I) und $\mathrm{P}_{\text {mean }} \geq 5,1 \mathrm{mbar}$ (Gruppe II) unterteilt. In Gruppe I betrug der $P_{\text {mean }} 4,3 \pm 0,2 \mathrm{mbar}$, in Gruppe II 6,0 $\pm 1,2 \mathrm{mbar}$ $(\mathrm{p}<0,001)$. Der VI lag in Gruppe I bei 0,6 $\pm 0,2$, in Gruppe II bei $1,3 \pm 0,5$ ( $p<0,001)$. In der Gesamtgruppe korrelierte der VI signifikant mit dem $P_{\text {mean }}(r: 0,66, p<0,001)$. 
Ziel der Therapie mit konstantem CPAP ist es, mit einem Druckniveau, das während einer manuellen Titration im Schlaflabor ermittelt wird, eine dauerhafte Behandlung des OSAS zu erreichen. Die Ergebnisse zur Effizienz der Therapie bestätigen, dass dieses Vorgehen bei zahlreichen Patienten eine suffiziente Behandlung ermöglicht [1]. Daneben sind automatische Therapieverfahren in der wissenschaftlichen Literatur akzeptiert [13]. Demgegenüber gibt es ein Kollektiv von Patienten, bei denen konstantes CPAP nicht effektiv ist oder die eine unzureichende Compliance zeigen $[2,3]$. Für diese Gruppe steht als alternative Behandlung die selbstadjustierende CPAP-Therapie zur Verfügung. Dabei hat sich das Verfahren auf der Basis der Messung der Impedanz der oberen Atemwege $\left(\mathrm{APAP}_{\mathrm{FOT}}\right)$ neben der Erfassung der Flusslimitation als wirksam erwiesen. Bei der Anwendung der automatischen Verfahren wurde eine ausgeprägte Variabilität des Druckbedarfes deutlich. Die Unterschiedlichkeit des Druckes nach Körperlage oder Schlafstadium während der einzelnen Nacht [16], aber auch im längerfristigen Verlauf, werden daher als eine weitere Indikation zur Auto-Therapie diskutiert. Ziel der vorliegenden Studie war es daher zu untersuchen, wie die Variabilität des Druckbedarfes zu quantifizieren ist und welche Bedeutung diese Phänomen in der klinischen Anwendung hat.

Bei den eingeschlossenen Patienten wurde die Indikation zur Therapie mit $\mathrm{APAP}_{\mathrm{FOT}}$ wegen Intoleranz oder Ineffektivität von konstantem CPAP gestellt. Unter $\mathrm{APAP}_{\mathrm{FOT}}$ zeigte sich eine befriedigende Beseitigung der respiratorischen Störungen mit Verbesserung des Schlafprofils. Beim Großteil der Patienten lag der VI in der 6-wöchigen Studie schon im Mittel oberhalb der Grenze von 0,75. Dies bedeutet, dass diese Gruppe (10 von 20 Untersuchten) regelhaft eine Schwankung des Druckes um mehr als 3 mbar zeigt. Bei diesen Patienten lag die Anzahl der Einzelnächte, in denen der Grenzwert überschritten wurde, zwischen 43 und $95 \%$. Dem stand eine Gruppe von 10 Personen gegenüber, bei denen der mittlere Wert den Grenzwert unterschritt. Unter diesen gab es jedoch nur drei, bei denen in weniger als $10 \%$ der Einzelnächte eine bedeutsame Variabilität nachzuweisen war. Insgesamt zeigte sich somit, dass bei $85 \%$ der Untersuchten eine relevante Variabilität nach mindestens einem Kriterium nachzuweisen war.

Das Ausmaß der Variabilität zeigte eine signifikante positive Korrelation mit dem mittleren Therapiedruck. Dies steht in Übereinstimmung mit früheren Untersuchungen, die gezeigt hatten, dass in 50\% der Zeit der minimal mögliche Druck und in $90 \%$ Drucke unter dem manuell titrierten Niveau appliziert wurden [7]. Daraus ergibt sich bei Patienten mit hohem mittleren Druck eine große Druckspanne, die für den VI entscheidend ist. Trotz dieser Abhängigkeit der über die Zeit gemittelten Werte fand sich auch bei den meisten Patienten mit niedrigem Druck eine bedeutsame Zahl von Einzelnächten, in denen der Grenzwert des VI überschritten wurde.

Die Bestimmung des VI wurde bereits früher beschrieben und hier in modifizierter Form durchgeführt [16]. Series et al. verglichen OSAS-Patienten, die sich durch das Fehlen oder Vorhandensein einer Lageabhängigkeit der respiratorischen Störungen unterschieden [16]. Die Autoren fanden, dass beim lageabhängigen OSAS der VI initial erhöht war, sich im weiteren Verlauf der Vergleichsgruppe annäherte. Dieses Phänomen konnte auch in un- serer Studie im Einzelfall gezeigt werden (Abb. $\mathbf{3 b}$ ). In der früheren Untersuchung fand sich ein erhöhter VI auch im nicht lageabhängigen Kollektiv.

Die Festlegung der Grenzwerte (mittlerer VI über sechs Wochen von $\geq 0,75$ oder mehr als $10 \%$ der Nächte $\geq 0,75$ ) erfolgte angelehnt an die Vorgaben zur Druckstabilität eines CPAP-Systems. Dabei werden Abweichungen vom eingestellten Wert um weniger als 0,5 mbar, entsprechend einer Spanne von 1 mbar verlangt. Dementsprechend wurden patientenbedingte Druckschwankungen in dieser Größe noch als stabiler Druckbedarf angesehen. Darüber hinaus können bei automatischen Systemen geringe, meist kurzfristige artefiziell bedingte Druckschwankungen beobachtet werden. Unter Berücksichtigung dieser Faktoren legten wir daher die Grenze auf eine Variationsbreite von 3 mbar fest, um nur klinische relevante Druckabweichungen zu erfassen. Trotz dieses permissiveren Vorgehens zeigte sich, dass eine bedeutsame Variabilität des Druckbedarfes keine Ausnahme, sondern die Regel in der einzelnen Behandlungsnacht und in der Dauerbehandlung darstellt. Die Ergebnisse bestätigten frühere Untersuchungen, nach denen trotz suffizienter Beseitigung der respiratorischen Störungen in annähernd $50 \%$ der Zeit das niedrigstmögliche Druckniveau, in $90 \%$ der Zeit ein Druckniveau unterhalb dem manuell titrierten Druck und in 5-10\% der Zeit ein Druck oberhalb des manuell titrierten Druckes notwendig war [7].

Limitationen der Studie ergeben sich aus dem untersuchten Kollektiv. Zwar repräsentieren die Patienten gut das Klientel unserer Klinik, allerdings waren Frauen nur unzureichend vertreten. Darüber hinaus wurden Patienten untersucht, bei denen die Therapie mit $\mathrm{APAP}_{\mathrm{FOT}}$ aus gezielten Indikationen (Intoleranz, Ineffektivität von CPAP) durchgeführt wurde. Daher können die Ergebnisse nicht unkritisch auf das unproblematische OSAS übertragen waren. Auch wenn frühere Untersuchungen mit $\mathrm{APAP}_{\mathrm{FOT}}$ die hohe Variabilität des Druckbedarfes auch bei unselektierten Patienten erwarten lassen [7 - 11], so sollte diese dennoch mit Hilfe des vorgestellten Verfahrens überprüft werden. Dabei könnte auch untersucht werden, ob Patienten mit hoher Variabilität gegenüber druckstabilen Patienten in somnologischen Messgrößen oder in der Compliance von automatischem CPAP profitieren. Auch wäre die Frage von Interesse, ob die Druckvariabilität sich zum Beispiel durch Maskenwechsel verändert.

Darüber hinaus muss diskutiert werden, dass unter den häuslichen Bedingungen eine polysomnographische Kontrolle der Ursachen der Druckschwankungen nicht erfolgen konnte. Die Effizienz des Systems wurde jedoch in zahlreichen Studien nachgewiesen [7-12]. Außerdem wurde eine Schwankungsbreite von 3 mbar als noch akzeptable Variabilität angesehen, auch um den Einfluss kurzfristiger artefizieller Druckvariationen aufzuheben.

Wir schließen, dass beim OSAS die Therapie mit einem fest eingestellten Therapiedruck nur in einzelnen Fällen dem Profil des Druckbedarfes der Patienten in adäquater Weise entspricht. 


\section{Literatur}

${ }^{1}$ Sullivan CE, Issa FG, Berthon-Jones $M$ et al. Reversal of obstructive sleep apnea by continuous positive airway pressure applied through the nares. Lancet 1981; 1 (8225): 862 - 865

2 Pepin JL, Krieger J, Rodenstein D et al. Effective compliance during the first 3 months of continuous positive airway pressure. A European prospective study of 121 patients. Am J Respir Crit Care Med 1999; 160: $1124-1129$

${ }^{3}$ Reeves-Hoché MK, Meck R, Zwillich CW. Nasal CPAP: an objective evaluation of patient compliance. Am J Respir Crit Care Med 1994; 149: $149-154$

${ }^{4}$ Berthon-Jones M, Lawrence S, Sullivan CE et al. Nasal continuous positive pressure treatment: current realities and future. Sleep 1996; 19: S131- 135

${ }^{5}$ Teschler H, Berthon-Jones M, Thompson AB et al. Automated continuous positive airway pressure titration for obstructive sleep apnea syndrome. Am J Respir Crit Care Med 1996; 154: 734- 740

${ }^{6}$ Teschler H, Farhat AA, Exner V et al. AutoSet nasal CPAP titration: constancy of pressure, compliance and effectiveness at 8 months followup. Eur Respir J 1997; 10: 2073-2078

${ }^{7}$ Randerath WJ, Parys K, Feldmeyer F et al. Self-adjusting nasal continuous positive airway pressure therapy based on measurement of impedance: A comparison of two different maximum pressure levels. Chest 1999; 116: 991 - 999

${ }^{8}$ Randerath W, Parys K, Lehmann D et al. Self-adjusting continuous positive airway pressure therapy based on the measurement of impe- dance. A comparison of free pressure variation and individually fixed higher minimum pressure. Respiration 2000; 67: 272 - 279

${ }^{9}$ Randerath WJ, Galetke W, David M et al. Prospective randomized comparison of impedance-controlled auto-continuous positive airway pressure $\left(\right.$ APAP $_{\mathrm{FOT}}$ ) with constant CPAP. Sleep Med 2001; 2: $115-124$

${ }^{10}$ Randerath WJ, Schraeder O, Galetke W et al. Autoadjusting cpap therapy based on impedance efficacy, compliance and acceptance. Am J Respir Crit Care Med 2001; 163: 652-657

${ }^{11}$ Ficker JH, Fuchs FS, Wiest GH et al. An auto-continuous positive airway pressure device controlled exclusively by the forced oscillation technique. Eur Respir J 2000; 16: 914-920

12 Randerath WJ, Galetke W, Feldmeyer F et al. Auto-adjusting CPAP based on impedance versus bilevel pressure in difficult-to-treat sleep apnea syndrome. A prospective randomized crossover study. Eur Respir J, 2002 Abstract im Druck,

${ }^{13}$ Berry RB, Parish JM, Hartse KM. The use of auto-titrating continuous positive airway pressure for treatment of adult obstructive sleep apnea. Sleep 2002; 25: 148 - 173

${ }^{14}$ Rechtschaffen A, Kales A. A manual of standardized terminology techniques and scoring system for sleep stages of human subjects. Los Angeles: Brain Information Service, University of California, 1968

15 ASDA Task Force. EEG arousals: scoring rules and examples. Sleep 1992; 15: $173-184$

${ }^{16}$ Series F, Marc I. Importance of sleep stage- and body position-dependence of sleep apnoea in determining benefits to auto-CPAP therapy. Eur Respir J 2001; 18: 170-175 